\title{
ARTICLE
}

\section{Vascular dementia: a pragmatic review}

\author{
Hugh Series \& Margaret Esiri
}

\begin{abstract}
Hugh Series is a consultant in the psychiatry of old age with Oxford Health NHS Foundation Trust and an honorary senior clinical lecturer at the University of Oxford. Margaret Esiri is Professor of Neuropathology at the University of Oxford. Correspondence Dr Hugh Series, South Locality CMHT for OIder Adults, Mental Health Building, Abingdon Hospital, Marcham Road, Abingdon, OX14 1AG, UK. Email: hugh.series@oxfordhealth.nhs.uk
\end{abstract}

\begin{abstract}
SUMMARY
Vascular dementia is associated with a group of diverse pathologies affecting the cerebrovascular circulation and with other dementia pathologies, particularly Alzheimer's disease. It is rather rare on its own. There is a spectrum of severity of cerebrovascular disease ranging from pathology but no cognitive impairment, to mild cognitive impairment to a dementia syndrome (vascular dementia). Where present, cerebrovascular disease can magnify the impact of other pathologies such as Alzheimer's disease. Current criteria for diagnosing vascular dementia are inadequate. Neuroimaging can be very helpful in defining the extent of pathology. Assessment needs to take into account a wide range of issues. Specific evidence-based treatments are limited, but attention should be given to managing risk factors and associated psychiatric problems such as depression.

\section{DECLARATION OF INTEREST}

M.E. receives financial support from the National Institute for Health Research through the Oxford Biomedical Research Centre.
\end{abstract}

Over the past 100 years, our understanding of dementia has undergone considerable change. For much of that time, 'senility' was seen as an almost inevitable concomitant of ageing, and was usually attributed to atherosclerosis. In the postwar period, a concept of multi-infarct dementia emerged (Hachinski 1974), but at the same time Alzheimer's disease came to be seen not as a rare early-onset disorder, but as the cause of most cases of dementia. Indeed, the concept of dementia itself was built around the impairments seen in Alzheimer's disease, notably the impairment of memory. With greater understanding of dementia, particularly its pathological basis, it came to be realised that many different kinds of pathology can produce a dementia syndrome, and that multiple types of pathology are the rule rather than the exception.

This article reviews our current understanding of how cerebrovascular disease contributes to cognitive impairment. Our aim is to help those involved in the assessment and management of people with cognitive impairments to understand the strengths and limitations of our current concepts, and to inform diagnosis and management. We try to tease apart the strands of clinical, imaging and pathological evidence about vascular dementia/vascular cognitive impairment in order to achieve improved clarity about the nature of the disease. We try to reach pragmatic conclusions, rather than provide a comprehensive account of a complex area of research.

\section{Vascular cognitive impairment}

Vascular cognitive impairment is a term which includes the full range of cognitive impairments that occur in people with cerebrovascular disease, ranging from mild cognitive impairment to vascular dementia (Erkinjuntti 2009). This is perhaps slightly confusing as the term mild cognitive impairment refers to a condition which, unlike vascular cognitive impairment, falls short of dementia in its severity.

The pathology of vascular dementia and vascular cognitive impairment is not well defined at present for several reasons. The clinical definitions of the two conditions are not well delineated and there are many different clinical schemes for assessing and diagnosing them. The clinicopathological correlation is not very good and varies from one series of cases to another. This is in part based on the source of cases, for example whether in the context of post-stroke cognitive performance or memory clinics. Cerebrovascular pathology itself is highly heterogeneous and there is a lack of clear agreement about how to characterise it and how different components relate to cognitive problems.

Some skewing of views has stemmed from the attempts, when arriving at definitions, to distinguish clearly between Alzheimer's disease and vascular dementia/vascular cognitive impairment despite the fact that many cases of dementia show both Alzheimer's disease pathology and vascular disease in the same brain. Some authors have suggested that it is no longer helpful to regard vascular dementia as a diagnostic category (Stewart 2002).

Another level of complexity in linking vascular pathology to dementia arises from the very 
important contribution from neuroimaging studies over the past 20-30 years. In some respects our understanding of the pathological basis of vascular dementia/vascular cognitive impairment now is at the same stage as our understanding of the pathological basis of Alzheimer's disease was in the early 1980s. In both conditions there are complex, distinct components to the pathology (plaques and tangles in Alzheimer's disease, different forms of vascular pathology in vascular dementia/vascular cognitive impairment) and we need to understand how these types of pathology are related and which are the key contributors to the dementia syndrome (Fig. 1). In Alzheimer's disease we now know, following detailed clinicopathological studies (Nagy 1995), that tangles correlate closely with the severity of dementia but we are at an earlier stage of discerning which of the forms of vascular pathology are the critical ones. We need this knowledge if we are to take steps to prevent vascular dementia/vascular cognitive impairment.

\section{Neuroradiology and pathology}

Many authorities advocate neuroimaging as part of the routine clinical work up recommended for people presenting with memory disorders. Why? What exactly do such studies tell us? We suggest four types of answer. First, neuroimaging may pick up surgically treatable conditions such as tumours, subdural haemorrhage or normal pressure hydrocephalus (although in practice the pick-up rate of treatable causes is very low indeed, even though relevant findings may be made). Second, it may help to improve the accuracy of diagnosis of the subtype of dementia, which may have implications for treatment. Third, it can be helpful for those affected and their families to see for themselves that the problems they struggle with have a real physical cause, and to feel that everything possible is being done to identify and treat the problem. In the UK, the National Health Service system for transmitting radiological images by internet (picture archiving and communications systems, PACS) allows doctors to show images to patients in the clinic, which can be a source of information and explanation, and a valuable aid to discussing diagnosis. For some people with limited insight into their condition it can help them to understand that there is a problem for which assistance might be beneficial. Finally, neuroimaging has a growing role in research, and there is reason to hope that it can assist in early diagnosis and disease monitoring.

As new treatments which may be effective in the early stages of disease are developed, it will

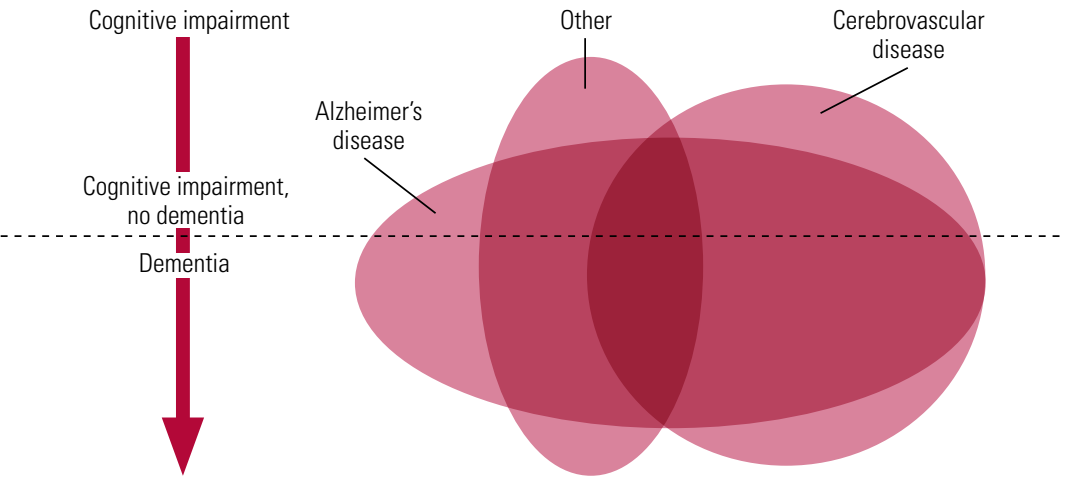

FIG 1

Schematic diagram showing the extensive coexistence of different types of pathology in many individuals and the correspondingly low frequency of pure forms of pathology. The degree of cognitive impairment increases from top to bottom of the diagram, and the horizontal line represents the point at which impairments are sufficiently great to be classified as dementia. 'Alzheimer's disease' and 'Cerebrovascular disease' indicate pathology, not necessarily with dementia syndrome. 'Other' includes many forms of pathology that can give rise to cognitive impairment, such as dementia with Lewy bodies.

become increasingly important to identify disease early. In the case of cerebrovascular disease, early identification of brain changes, perhaps even before clinical signs of dementia, may mean that there is stronger motivation to manage risk factors at a relatively early stage. Neuroradiology has reached a level of development where some authors have argued that neuroradiology rather than pathology should be considered the gold standard against which new diagnostic criteria should be judged (Moorhouse 2008).

Space constraints do not allow us to discuss here the wide variety of new imaging techniques now available (see Barkhof 2011). Instead, we will focus on computerised tomography (CT) and magnetic resonance imaging (MRI) and on the pathology that these imaging techniques reveal.

\section{Forms of pathology that may contribute to vascular dementia/vascular cognitive impairment}

\section{Major stroke}

Large vessel disease includes single and multiple infarcts that can be readily seen by CT or MRI. They can be found in main artery territories, or in the watershed zones between the main arterial territories. Vascular dementia can be caused by multiple or single strategic infarcts (i.e. in locations critical for cognitive function such as thalamus). The National Institute of Neurological Disorders and Stroke and the Association Internationale pour la Recherche et l'Enseignement en Neurosciences (NINDS-AIREN) criteria (Box 1) specify the brain regions in which infarcts indicate a diagnosis of vascular dementia. For large vessel 
BOX 1 NINDS-AIREN criteria for the diagnosis of vascular dementia

I Probable vascular dementia:

1 dementia syndrome not due to delirium, psychosis, aphasia or sensorimotor impairment, and

2 cerebrovascular disease defined by presence of focal neurological signs and evidence of relevant cerebrovascular disease by brain imaging (further specified), and

3 a relationship between 1 and 2 such as dementia occurring within 3 months of a stroke, or abrupt deterioration, or fluctuating stepwise progression.

II Features consistent with probable vascular dementia include early gait disturbance, frequent falls, early urinary symptoms, pseudobulbar palsy, personality and mood changes, subcortical deficits such as psychomotor retardation and abnormal executive function.
III Features making vascular dementia unlikely include clinical symptoms in the absence of focal neurological signs or cerebrovascular lesions on brain CT or MRI. diagnosed in the absence of brain imaging studies or clear temporal relationship of dementia to stroke.

$\checkmark$ Definite vascular dementia requires clinical criteria for probable vascular dementia and pathological evidence of cerebrovascular disease in the absence of tangles and plaques or other types of dementia pathology.

The term 'Alzheimer's disease with cerebrovascular disease' refers to patients fulfilling criteria for possible Alzheimer's disease who also have clinical or imaging evidence of cerebrovascular disease. Avoid the term 'mixed dementia'.

(After Román 1993
IV Possible vascular dementia may be

clinically silent, but may be accompanied by a history of transient ischaemic attack or stroke. Lacunar infarcts in thalamus can cause prominent cognitive problems. At least two lacunes are required for a diagnosis of vascular dementia. There is good evidence that lacunar infarcts can form a substrate for vascular dementia. They are often multiple and bilateral and are associated with disease affecting the small arteries and arterioles subserving deep grey and white matter (Fig. 2).

\section{Microinfarcts}

These are ischaemic lesions that are too small to be seen with the naked eye and are detected when microscopic histology is carried out. They can occur in the subcortical tissue but are most frequently found in cortex. They can result from a number of different disease processes in vessel walls (e.g. vasculitis), but most are probably due to embolic occlusion of small arteries by microemboli.

\section{Diffuse subcortical small vessel disease}

This is damage to the small subcortical vessels that supply deep grey matter and white matter.

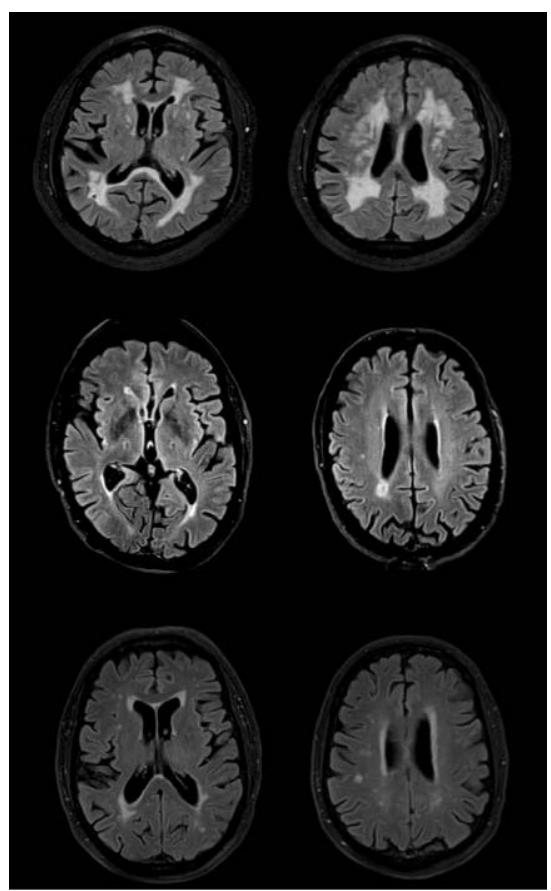

infarcts to be sufficient for diagnosis of vascular dementia they should be either bilateral or in the dominant hemisphere.

Studies of patients after they have had an ischaemic stroke show that progressive dementia is significantly more common than in an age- and gender-matched control population. Thus, in a study of 251 patients aged over 60 years, Tatemichi et al (1992) found dementia in $26 \%$ of patients 3 months after they had had a stroke compared with $3.2 \%$ in a control sample. A meta-analysis of dementia after stroke (Pendlebury 2009) found that in $10 \%$ of patients, dementia was present before the stroke occurred and in a further 10\% there was evidence of dementia developing after one stroke, and rose to more than 30\% in those who had had more than one stroke. This points to the significant contribution that major ischaemic stroke can make to dementia. A deficiency in most of the literature examining the links between stroke and subsequent dementia is that postmortem studies have not been performed so that the relative contributions of Alzheimer's disease pathology, perhaps 'unmasked' by vascular disease, and the vascular disease itself were not assessed.

\section{Lacunar stroke}

Lacunes are small, round or oval subcortical infarcts from 3 to $20 \mathrm{~mm}$ in size. They may be
Magnetic resonance images of vascular damage: (a) 81-year-old patient (MMSE $=21 / 30$ ) with dementia and diffuse white matter changes; (b) 76-year-old patient (MMSE $=20 / 30$ ) with bilateral lacunar infarcts; (c) 77-year-old cognitively healthy control (MMSE $=30 / 30$ ) with mild white matter changes. Images were acquired with a FLAIR sequence in a 3T scanner. 
The vessels affected are small arteries and arterioles that branch from proximal parts of the major cerebral arteries to supply the basal ganglia and thalamus, and penetrating arterioles from the pial surface that reach through the cerebral cortex to supply the white matter. The perivascular Virchow-Robin spaces around these vessels are frequently widened, a process referred to as cribriform change. The tissue supplied by these small vessels may suffer from lacunar infarction or hypoperfusion of blood that renders it damaged but not dead. Radiological changes of small vessel disease are very commonly seen in scans of older people, and small vessel disease may even account for the largest proportion of vascular dementia (van der Flier 2009). Erkinjuntti et al (2000) have proposed specific radiological criteria for subcortical ischaemic vascular dementia.

A number of types of small vessel disease are seen on imaging (Fig. 2). White matter hyperintensities appear bright on $T_{2}$-weighted and FLAIR MRI images. When seen in CT, they appear dark and are referred to as hypodense areas or leukoaraiosis. They become more common with increasing age and are associated with cognitive impairment. As they are so commonly seen, the NINDS-AIREN criteria set an arbitrary cutoff that at least $25 \%$ of the total white matter should be affected for a diagnosis of vascular dementia. White matter hyperintensities can be seen around the lateral ventricles, often starting at the poles, and in subcortical areas, although these two types of distribution are thought to have different aetiologies. They are often rated either by eye or by using an automated process as mild (punctiform), moderate (beginning confluent) or severe (confluent) (Scheltens 2000).

An unusual form of subcortical small vessel disease occurs in the rare inherited condition of CADASIL (cerebral autosomal dominant arteriopathy with subcortical infarcts and leukoencephalopathy) in which a mutation in the Notch3 gene results in deposition of granular material in the walls of small arteries and arterioles, narrowing of vessel lumens and destruction of smooth muscle cells in their walls.

\section{Cerebral amyloid (congophilic) angiopathy}

This term refers to the deposition of abnormal protein in the form of twisted beta-pleated sheets in the walls of small arteries and arterioles where it eventually replaces the smooth muscle. It principally affects the leptomeningeal and cortical vessels. In most cases the protein is the amyloid beta protein that also forms the characteristic amyloid plaques of Alzheimer's disease. Cerebral amyloid angiopathy occurs to a variable extent in almost all cases of Alzheimer's disease. However, it also occurs in about a third of elderly individuals that do not meet criteria for pathological Alzheimer's disease and in these cases it can be shown to contribute to vascular dementia or vascular cognitive impairment (Esiri 1997). A key feature of cerebral amyloid angiopathy is microbleeds which are small dots best seen in $T_{2}{ }^{*}$-weighted images. They represent leakage of hemosiderin from damaged small blood vessels and, although present at low levels in community populations, are much more common in stroke and vascular dementia populations.

\section{Mixed or multiple forms of vascular pathology}

It is commonly the case that in an elderly brain there is more than one of these forms of vascular pathology and it can be difficult to define the exact component of mixed pathology that contributes most to vascular dementia or vascular cognitive impairment. In the unselected communitybased population of elderly participants that contributed to the Medical Research Council Cognitive Function and Ageing Study, it was only in cases that had more than one form of vascular pathology that vascular disease contributed to dementia (Ince 2001). However, it is noteworthy that in almost all cases one form of vascular pathology was small vessel disease. Thus, there is good reason to believe that small vessel disease, including lacunes that it gives rise to, is a particularly important contributor to vascular dementia/vascular cognitive impairment.

\section{What is the mechanism by which vascular pathology causes vascular dementia/ vascular cognitive impairment?}

Given the importance of small vessel disease in vascular dementia and vascular cognitive impairment, it is reasonable to suggest that cognitive dysfunction results at least in part from interruption of axonal connections between one part of the cerebral cortex and another, and between the cerebral cortex and deep grey matter. Small vessel disease affects particularly frontal lobe white matter and the closely related basal ganglia, so it is not surprising that the cognitive dysfunction commonly seen in vascular dementia involves executive activity which is known to be a function of the frontal lobe (see below). It is interesting that in CADASIL the cognitive dysfunction closely resembles that seen in elderly sporadic cases of vascular dementia/vascular cognitive impairment (Charlton 2006). Cerebral amyloid angiopathy may also affect white matter 
function because this is the final destination for the blood flowing in the cortical arterioles affected by this condition. In macroinfarction, lacunes and microinfarction, it is the loss of neurons that is thought to be important and there is evidence that after a stroke, dementia is more likely if the stroke was severe, therefore destroying more neurons.

\section{Diagnostic criteria}

At least eight different sets of diagnostic criteria for vascular dementia have been published, including the Hachinski Ischaemic Scale, DSM-IV (and its predecessors), ICD-10 (and its predecessors), and criteria from the State of California's Alzheimer's Disease Diagnostic and Treatment Centers (ADDTC) and from NINDS-AIREN (Román 1993; Wiederkehr 2008; Box 1). To determine how effective these criteria are at identifying patients with vascular dementia in life, Wiederkehr et al (2008) conducted a literature search to identify studies which compared lifetime diagnoses using one or other of these criteria against post-mortem pathological diagnoses. Only six studies were identified. Sensitivities across the studies ranged from 20 to $100 \%$, mostly in the range $20-60 \%$; specificities ranged from 13 to $100 \%$, mostly in the range $60-90 \%$. The NINDS-AIREN criteria tended to have the highest specificity, although lower sensitivity. Sensitivities were usually lower than specificities. One problem with the use of sensitivity and specificity is that they are affected by the prevalence of the disorder, and to avoid this problem, likelihood ratios may be calculated. A positive likelihood ratio is the odds in favour of the disease being present, and a negative likelihood ratio is the odds against this. On the basis of standard criteria, Wiederkehr et al found that, taking a positive likelihood ratio $>10$ and a negative likelihood ratio $<0.1$ as the threshold of acceptability for a diagnostic test, only the NINDS-AIREN criteria reached this threshold, with ADDTC coming close in one study.

In two studies (Fischer 1991; Rockwood 2003) comparing the published criteria against clinical judgement, DSM-IV criteria were found to be the most sensitive, whereas the NINDS-AIREN criteria were the most specific. In another comparative study, Verhey et al (1996) found that all of seven sets of criteria agreed on only 8 out of 124 cases of dementia.

Thus, even in research centres it is difficult to achieve accurate diagnoses in life. Among the factors that contribute to the difficulty are issues regarding the diagnosis of the dementia syndrome: that it may not be appropriate to base this on definitions of dementia derived largely from Alzheimer's disease, the heterogeneity of pathology in cerebrovascular disease, and the lack of agreement on pathological criteria for diagnosis. More research is needed to determine which of the many pathological cerebrovascular processes contribute to vascular cognitive impairment and in what way.

Most diagnostic criteria specify that there must be a dementia syndrome and then set out criteria to classify it as cerebrovascular in origin. However, as Alzheimer's disease is the predominant form of dementia, this can dominate the clinical concept of the dementia syndrome, for example by emphasising the memory aspect. Studies comparing cognitive changes in Alzheimer's disease with those in vascular dementia (O'Brien 2003; Sachdev 2003) have found that for similar levels of cognitive deficit, patients with vascular dementia were more likely to have relatively better preserved verbal episodic memory and poorer frontal executive functioning. That there should be differences is not surprising in view of the different pathologies and the tendency of Alzheimer's pathology to start in hippocampal areas involved in processing memories. However, not all studies have confirmed that vascular cognitive impairment does affect executive functioning in such a distinct way (Price 2005).

There is widespread awareness that current criteria and definitions are an inadequate basis for the research that is necessary to resolve some of these complex issues, and so an expert group has proposed a set of harmonisation standards for the collection of research data in this area (Hachinski 2006). These do not constitute new diagnostic criteria, but may inform their development.

\section{Epidemiology}

The difficulties referred to above in agreeing clinical criteria for diagnosis and the variability of sources of cases mean that epidemiological studies also lack agreement. In population-based studies, estimates of the prevalence of vascular dementia in Europe and North America vary between 1.2 and $4.2 \%$ in individuals over the age of 65 years (Hebert 1995). In Japan they are higher (Ueda 1992; Ikeda 2001). The prevalence and incidence of vascular dementia increases with age over the age of 65 years. Thus, in a major European study, incidence rose from 0.7 per 1000 person years at 65-69 years to 8.1 per 1000 person years at $90+$ years (Fratiglioni 2000). However, this is much less steep a rise with age than for Alzheimer's disease and pathological studies on selected populations suggest that the frequency of pure 
vascular dementia is actually considerably lower than these epidemiological studies indicate.

Risk factors for vascular dementia include hypertension (although the risk is less than that for stroke and Alzheimer's disease, and it was only a risk factor for females in a Canadian study (Hebert 2000)), orthostatic hypotension, cardiac disease, diabetes, smoking, obesity, major surgery, elevated blood homocysteine level and hyperlipidaemia.

\section{Clinical assessment}

The goals of clinical assessment in vascular cognitive impairment include diagnosis and identification of specific therapeutic targets in each patient (Box 2). It is clear that many patients have more than one pathological process present. Cerebrovascular disease is common, so clinicians should have a low threshold for considering that it may be present and contributing to the presentation, even if another pathological process, such as Alzheimer's disease, has also been identified (Lopez 2009). A useful resource is the list of assessment items in the vascular cognitive impairment harmonisation standards developed by NINDS and the Canadian Stroke Network
(Hachinski 2006). However, these are intended for assessing individuals in research studies and they are perhaps overinclusive in clinical settings. For a full account of assessment procedures see Wilcock et al (1999).

\section{Treatment}

\section{Treatment of cognitive changes}

Two randomised controlled trials have examined the efficacy of donepezil in vascular dementia (Wilkinson 2003, 2005; Black 2003) and found only modest efficacy. A trial of galantamine in patients with mixed Alzheimer's disease with cerebrovascular disease found small benefit only (Erkinjuntti 2002), although benefit was seen in the subgroup with probable vascular dementia. Two randomised controlled studies have tested the effects of memantine in vascular dementia and both found only modest efficacy (Orgogozo 2002; Wilcock 2002). Neither memantine nor any of the cholinesterase inhibitors is licensed in vascular dementia, and the National Institute for Health and Clinical Excellence (NICE) does not recommend their use for this indication (National Institute for Health and Clinical Excellence 2011b).

BOX 2 Clinical assessment

\section{History}

Time and mode of onset of cognitive impairment, relation to stroke, transient ischaemic attack myocardial infarction. Full health history, diabetes, hypertension, hyperlipidaemia. Alcohol, drugs, smoking. Family history of cardiovascular or neurological disease.

\section{Collateral history}

Confirm extent of functional loss (this is required to diagnose dementia syndrome).

\section{Physical examination}

Focal neurological signs, asymmetries of power, tone, reflexes and sensation, tremor, and balance and gait abnormalities. Cardiovascular system, signs of relevant systemic disease such as diabetes mellitus. Other associated degenerative disorders such as Parkinson's disease. Extrapyramidal signs could be a feature of cerebrovascular disease, but also of Parkinson's disease, progressive supranuclear palsy, other dementias, or neurological disorders such as multiple sclerosis. 'Classic' physical signs associated with vascular dementia include dysarthria, pseudobulbar palsy, gait abnormalities and emotional lability, but it is doubtful how far they positively support a diagnosis of vascular dementia.

\section{Psychiatric disorders}

Depression may affect the diagnostic assessment and require treatment in its own right. Psychosis, whether chronic or acute, can produce cognitive impairment.

\section{Investigations}

Blood screen, including vitamin $B_{12}$ and folate. Growing evidence that homocysteine is a risk factor for dementia, and may prove to be a useful treatment target (Smith 2010). The National Institute for Health and Clinical Excellence (NICE; 2011a) does not recommend routine screening for syphilis or HIV unless the history is suggestive. Chest $X$-ray and electrocardiogram should be considered, if relevant, but electroencephalogram should be reserved for those who have seizures or in whom Creutzfeldt-Jakob disease or frontotemporal dementia is possible. Cerebrospinal fluid analysis is not widely used in UK clinical practice and not recommended by NICE, but it is more common in Scandinavia. Neuroimaging is recommended. Computed tomography is widely available, economical and helpful, but MRI provides more information, although it is more expensive and may be less well tolerated because each MRI scan takes much longer (20-40 min) than CT and the person has to lie still with the whole body inside the scanner. Many other types of imaging, notably single-photon emission computed tomography or positron emission tomography to investigate regional blood flow, are useful. It is beyond the scope of this article to recommend particular types of scan, but the closer the dialogue between the requesting clinician and the radiologist, the more appropriate the method of scanning is likely to be, and the more informative the report. It may be helpful to agree a 'dementia protocol' with the neuroradiology department in advance, and any opportunity for joint clinicoradiological meetings will enhance the effective use of neuroimaging.

\section{Neuropsychological testing}

To establish breadth and depth of cognitive impairment. Sometimes the diagnosis will be apparent with only very limited testing such as with the Mini-Mental State Examination (Folstein 1975), but in cerebrovascular disease there may well be significant breadth of impairment requiring more extensive testing. Hachinski et al (2006) recommended a 5-minute screening test based on subscales of the Montreal Cognitive Assessment (available free of charge at www.mocatest.org) as a quick and useful instrument that can be used by telephone if necessary. Screening tests for depression such as the Cornell Scale for Depression in Dementia (Alexopoulos 1988) may be useful. 
Other agents have been tested: calcium channel blockers (e.g. nimodipine), nootropics (e.g. piracetam, citicoline), xanthenes (e.g. pentoxifylline), vasodilators, ergot derivatives and antithrombotics (e.g. aspirin, gingko). Most have not been shown to have any effect and none has more than modest effects (for reviews see Erkinjuntti et al 2004 and Román 2005).

\section{Symptomatic treatment}

Depression is common and requires treatment, whether or not there is any aetiological relation to cognitive impairment. There is an emerging concept of vascular depression (Alexopoulos 1997), and although attempts to treat it are appropriate, treatment may be less successful than in other types of depression (Sheline 2010). Depression is a risk factor for dementia.

Psychosis may also be present and may require treatment in its own right, although clinicians need to be mindful of the evidence that antipsychotic medication may increase cerebrovascular mortality in people with dementia and should note the advice to avoid antipsychotics if possible (Banerjee 2009).

As in any form of dementia, appropriate support and information for both patients and carers is essential. These arguably contribute more to well-being than currently available drug treatments.

\section{Control of vascular risk factors}

There is no shortage of potential targets for the management of vascular cognitive impairment risk factors, but perhaps surprisingly the evidence that this reduces rates of onset or progression is limited. A Cochrane review failed to find convincing evidence that lowering blood pressure prevents the development of dementia or cognitive impairment in patients with hypertension without prior cerebrovascular disease (McGuinness 2009), although the Syst-Eur and PROGRESS studies showed small but significant reductions in dementia from treating hypertension (Forette 2002; Tzourio 2003).

Several studies have failed to show a benefit of statins on vascular dementia, which Bowler (2007) suggests may be because subcortical vascular cognitive impairment is the most common form of vascular cognitive impairment and hypertension is its strongest treatable risk factor, whereas cholesterol has little association with small vessel disease. Nevertheless, most clinicians would consider it appropriate to treat vascular risk factors in any case.
B0X 3 Key learning points

- Vascular dementia is not a single entity but any form of dementia arising from a wide variety of impairments in the cerebrovascular circulation

- Not everyone with evidence of cerebrovascular disease has a cognitive impairment

- The term vascular cognitive impairment includes vascular dementia, but also mild cognitive impairment of vascular origin

- Even in research centres, the ability to diagnose vascular dementia in life as indicated by post-mortem pathology later is limited

- Pure vascular dementia is rare: vascular dementia is most commonly associated with Alzheimer's disease

- Treatment of vascular risk factors is appropriate, although the evidence base is small

- Cholinesterase inhibitors have only a small benefit and are not licensed for use in vascular dementia

This review has sought to offer a pragmatic account of the complexities of vascular dementia, and its key learning points are summarised in Box 3 .

\section{Acknowledgement}

We are extremely grateful to Dr Giovanna Zamboni for providing the images in Fig. 2.

\section{References}

Alexopoulos GS, Abrams RC, Young RC, et al (1988) Cornell Scale for Depression in Dementia. Biological Psychiatry 23: 271-84.

Alexopoulos GS, Meyers BS, Young RC, et al (1997) 'Vascular depression' hypothesis. Archives of General Psychiatry 54: 915-22.

Banerjee S (2009) The Use of Antipsychotic Medication for People with Dementia: Time for Action. Department of Health.

Barkhof F, Fox NC, Bastos-Leite AJ, et al (2011) Neuroimaging in Dementia. Springer

Black S, Roman GC, Geldmacher DS, et al (2003) Efficacy and tolerability of donepezil in vascular dementia: positive results of a 24-week, multicenter, international, randomized, placebo-controlled clinical trial. Stroke 34: 2323-32.

Bowler JV (2007) Modern concept of vascular cognitive impairment. British Medical Bulletin 83: 291-305.

Charlton RA, Morris RG, Nitkunan A, et al (2006) The cognitive profiles of CADASIL and sporadic small vessel disease. Neurology 66: 1523.

Erkinjuntti T, Inzitari D, Pantoni L, et al (2000) Research criteria for subcortical vascular dementia in clinical trials. Journal of Neural Transmission 59 (suppl): 23-30.

Erkinjuntti T, Kurz A, Gauthier S, et al (2002) Efficacy of galantamine in probable vascular dementia and Alzheimer's disease combined with cerebrovascular disease: a randomised trial. Lancet 359: 128390.

Erkinjuntti T, Roman G, Gauthier S, et al (2004) Emerging therapies for vascular dementia and vascular cognitive impairment. Stroke 35 : 1010. 
Erkinjuntti T, Gauthier S (2009) Diagnosing vascular cognitive impairment and dementia: concepts and controversies. In Vascular Cognitive Impairment in Clinical Practice (eds LO Wahlund, T Erkinjuntti, S Gauthier): 3-9. Cambridge University Press.

Esiri MM, Wilcock GK, Morris JH (1997) Neuropathological assessment of the lesions of significance in vascular dementia. Journal of Neurology, Neurosurgery \& Psychiatry 63: 749.

Fischer P, Jellinger K, Gatterer G, et al (1991) Prospective neuropathological validation of Hachinski's Ischaemic Score in dementias. Journal of Neurology, Neurosurgery \& Psychiatry 54: 580.

Folstein MF, Folstein SE, McHugh PR (1975) 'Mini-mental state'. A practical method for grading the cognitive state of patients for the clinician. Journal of Psychiatric Research 12: 189-98.

Forette F, Seux ML, Staessen JA, et al (2002) The prevention of dementia with antihypertensive treatment: new evidence from the Systolic Hypertension in Europe (Syst-Eur) study. Archives of Internal Medicine 162: 2046-52.

Fratiglioni L, Launer LJ, Andersen K, et al (2000) Incidence of dementia and major subtypes in Europe: a collaborative study of populationbased cohorts. Neurology 54: S10-5.

Hachinski VC, Lassen NA, Marshall J (1974) Multi-infarct dementia: a cause of mental deterioration in the elderly. Lancet 304: 207-9.

Hachinski V, ladecola C, Petersen RC, et al (2006) National Institute of Neurological Disorders and Stroke-Canadian Stroke Network vascular cognitive impairment harmonization standards. Stroke 37: 2220-41.

Hebert R, Brayne C (1995) Epidemiology of vascular dementia. Neuroepidemiology 14: 240-57.

Hebert R, Lindsay J, Verreault R, et al (2000) Vascular dementia: incidence and risk factors in the Canadian study of health and aging. Stroke 31: 1487.

Ikeda M, Hokoishi K, Maki N, et al (2001) Increased prevalence of vascular dementia in Japan. Neurology 57: 839.

Ince G (2001) Pathological correlates of late-onset dementia in a multicentre, community-based population in England and Wales. Lancet 357: 169-75.

Lopez OL, Wolk DA (2009) Clinical evaluation: a systematic but user-friendly approach. In Vascular Cognitive Impairment in Clinical Practice (eds LO Wahlund, T Erkinjuntti, S Gauthier): 32-45. Cambridge University Press.

McGuinness B, Todd S, Passmore P, et al (2009) Blood pressure lowering in patients without prior cerebrovascular disease for prevention of cognitive impairment and dementia. Cochrane Database of Systematic Reviews issue 4: CD004034 (doi: 10.1002/14651858. CD004034.pub3).

Moorhouse P, Rockwood K (2008) Vascular cognitive impairment: current concepts and clinical developments. Lancet Neurology 7 : $246-55$

Nagy ZS, Esiri MM, Jobst KA, et al (1995) Relative roles of plaques and tangles in the dementia of Alzheimer's disease: correlations using three sets of neuropathological criteria. Dementia and Geriatric Cognitive Disorders 6: 21-31.

National Institute for Health and Clinical Excellence (2011a) Dementia: Supporting People with Dementia and their Carers in Health and Social Care (Clinical Guideline 42) (amended March 2011). NICE.

National Institute for Health and Clinical Excellence (2011b) Alzheimer's Disease - Donepezil, Galantamine, Rivastigmine and Memantine (Technology Appraisal 217). NICE.

O'Brien JT, Erkinjuntti T, Reisberg B, et al (2003) Vascular cognitive impairment. Lancet Neurology 2: 89-98.

Orgogozo J, Rigaud A, Stoffler A, et al (2002) Efficacy and safety of memantine in patients with mild to moderate vascular dementia: a randomized, placebo-controlled trial (MMM 300). Stroke 33: 1834-9.
Pendlebury ST, Rothwell PM (2009) Prevalence, incidence, and factors associated with pre-stroke and post-stroke dementia: a systematic review and meta-analysis. Lancet Neurology 8: 1006-18.

Price CC, Jefferson AL, Merino JG, et al (2005) Subcortical vascular dementia. Neurology 65: 376-82.

Rockwood K, Davis H, MacKnight C, et al (2003) The Consortium to Investigate Vascular Impairment of Cognition: methods and first findings. Canadian Journal of Neurological Sciences 30: 237-43.

Román GC, Tatemichi TK, Erkinjuntti T, et al (1993) Vascular dementia: diagnostic criteria for research studies. Report of the NINDS-AIREN International Workshop. Neurology 43: 250-60.

Román G (2005) Therapeutic strategies for vascular dementia. In Dementia (3rd edn) (eds A Burns, J 0'Brien, D Ames): 574-600. Hodder Arnold.

Sachdev PS, Looi JCL (2003) Neuropsychological differentiation of Alzheimer's disease and vascular dementia. In Vascular Cognitive Impairment: Preventable Dementia (eds JV Bowler, V Hachinski): 152-76. Oxford University Press.

Scheltens P, Erkinjuntti T, Leys D, et al (2000) White matter changes on CT and MRI: an overview of visual rating scales. European Neurology 39: 80-9.

Sheline YI, Pieper CF, Barch DM, et al (2010) Support for the vascular depression hypothesis in late-life depression: results of a 2-site, prospective, antidepressant treatment trial. Archives of General Psychiatry 67: 277-85.

Smith AD, Smith SM, de Jager CA, et al (2010) Homocysteinelowering by $B$ vitamins slows the rate of accelerated brain atrophy in mild cognitive impairment: a randomized controlled trial. PloS One 5: e12244.

Stewart R (2002) Vascular dementia: a diagnosis running out of time. British Journal of Psychiatry 180: 152-6.

Tatemichi TK, Desmond DW, Mayeux R, et al (1992) Dementia after stroke. Neurology 42: 1185.

Tzourio C, Anderson C (2003) Effects of blood pressure lowering with perindopril and indapamide therapy on dementia and cognitive decline in patients with cerebrovascular disease. Archives of Internal Medicine 163: $1069-75$

Ueda K, Kawano H, Hasuo Y, et al (1992) Prevalence and etiology of dementia in a Japanese community. Stroke 23: 798.

van der Flier W, Staekenborg SS, Barkhof F, et al (2009) Structural neuroimaging: CT and MRI. In Vascular Cognitive Impairment in Clinical Practice (eds LO Wahlund, T Erkinjuntti, S Gauthier): 58-69. Cambridge University Press.

Verhey FRJ, Lodder J, Rozendaal N, et al (1996) Comparison of seven sets of criteria used for the diagnosis of vascular dementia. Neuroepidemiology 15: 166-72.

Wiederkehr S, Simard M, Fortin C, et al (2008) Validity of the clinical diagnostic criteria for vascular dementia: a critical review. Part II. Journal of Neuropsychiatry and Clinical Neuroscience 20: 162-77.

Wilcock GK, Bucks RS, Rockwood K (1999) Diagnosis and Management of Dementia: A Manual for Memory Disorders Teams. Oxford University Press.

Wilcock G, Möbius HJ, Stöffler A (2002) A double-blind, placebocontrolled multicentre study of memantine in mild to moderate vascular dementia (MMM500). International Clinical Psychopharmacology 17 : 297-305.

Wilkinson D, Doody R, Helme R, et al (2003) Donepezil in vascular dementia: a randomized, placebo-controlled study. Neurology 61: 479-86

Wilkinson DG, Doody RS, Black SE, et al (2005) Donepezil in vascular dementia: combined analysis of two large-scale clinical trials. Dementia and Geriatric Cognitive Disorders 20: 338-44.

\section{MCO answers}

$1 d \quad 2$ e $3 c \quad 4 b \quad 5 a$ 
MCOs

Select the single best option for each question stem

1 Appearances on an MRI scan that are not likely to be a normal expression of ageing include:

a generalised atrophy

b a single lacunar infarct

c punctiform white matter hyperintensities

d severe cribriform state (état criblé)

e periventricular white matter hyperintensities.

2 Drugs licensed for use in vascular dementia include:
a donepezil
b aspirin
c galantamine
d piracetam
e none of the above.

3 Vascular dementia:

a is more severe than vascular cognitive impairment

$\mathrm{b}$ is the same as multi-infarct dementia

c is often present in people with Alzheimer's disease

d can be prevented by statins

$\mathrm{e}$ is rarely associated with depression.

4 Research shows that:

a people with moderate to severe cognitive impairment rarely show Alzheimer-type pathology

b mixed Alzheimer's disease/cerebrovascular disease pathology is more common than pure cerebrovascular disease

c cerebrovascular disease results from a single pathological process d a finding of any cerebrovascular disease on neuroimaging demonstrates that dementia is vascular in origin

e mild white matter change on MRI is an abnormal finding in vascular dementia.

5 According to the literature:

a published criteria for vascular dementia show a relatively low level of agreement

b neuroimaging does not add useful information in making a diagnosis of vascular dementia

c reducing blood cholesterol has been shown to reduce the risk of developing vascular dementia

$\mathrm{d}$ depression is not a risk factor for dementia

e cribriform change refers to narrowed VirchowRobin spaces around blood vessels. 